\title{
A clinical pharmacology study of the novel, selective urate reabsorption inhibitor dotinurad in outpatients
}

\author{
Tatsuo Hosoya ${ }^{1}$ Kazuki Furuno ${ }^{2} \cdot$ Shingo Kanda ${ }^{2}$
}

Received: 17 September 2019 / Accepted: 23 January 2020 / Published online: 18 February 2020

(c) The Author(s) 2020

\begin{abstract}
Background Dotinurad is a novel, selective urate reabsorption inhibitor (SURI), which reduces serum uric acid levels by selective inhibition of the urate transporter 1 (URAT1). The Japanese guideline for the management of hyperuricemia and gout recommends that drug selection should be based on classification of hyperuricemia as a fundamental principle. However, there may be some cases where this principle is not observed. We investigated the pharmacodynamics and safety of dotinurad in outpatients with uric acid overproduction or uric acid underexcretion type.

Methods This was a multicenter, open-label, forced titration study. Patients were classified as uric acid overproduction or underexcretion type. Study treatment was initiated at $0.5 \mathrm{mg} /$ day, followed by dose titration to the estimated maximum dose of $4 \mathrm{mg} /$ day over 14 weeks. The primary endpoint was urinary uric acid excretion at each 24-h urine collection.

Results A total of 26 hyperuricemic patients with or without gout were enrolled in the study and assigned to the uric acid overproduction group (overproduction group) or the uric acid underexcretion group (underexcretion group). Although urinary uric acid excretion, the primary endpoint, tended to be slightly greater in the overproduction group, no notable difference was noted between the two hyperuricemic types. Neither type had noteworthy safety concerns associated with dotinurad. Conclusion The results of the study demonstrated no relevant differences between the hyperuricemic types in terms of pharmacodynamic action and safety of dotinurad.
\end{abstract}

Keywords Hyperuricemia $\cdot$ Gout $\cdot$ Selective urate reabsorption inhibitor (SURI) · URAT1 inhibitor · Dotinurad · FYU-981

\section{Introduction}

Hyperuricemia is considered to be a pathological condition causing uric acid deposition diseases (e.g., gouty arthritis, tophus), and is defined by serum uric acid levels exceeding $7.0 \mathrm{mg} / \mathrm{dL}[1,2]$. Urinary calculi constitute another uric acid deposition disease. It is common knowledge that the pathological condition of hyperuricosuria facilitates calculus

Electronic supplementary material The online version of this article (https://doi.org/10.1007/s10157-020-01857-0) contains supplementary material, which is available to authorized users.

Kazuki Furuno

kazuki.furuno@mochida.co.jp

1 Jikei University School of Medicine, 3-25-8, Nishi-Shimbashi, Minato-ku, Tokyo 105-8461, Japan

2 Clinical Research Department, Mochida Pharmaceutical Co., Ltd., 1-22 Yotsuya, Shinjuku-ku, Tokyo 160-0004, Japan formation [3] and a formed calculus lodged in the urinary tract often causes severe pain. Calculi are known to be associated with elevated serum creatinine levels and decreased estimated glomerular filtration rate (eGFR), and may affect renal function [4]. Correcting the hyperuricemic condition and maintaining uric acid levels $\leq 6.0 \mathrm{mg} / \mathrm{dL}$ (the treatment goal) are critical for the prevention of uric acid deposition diseases [1].

This study was planned and conducted in accordance with the Japanese guideline for the management of hyperuricemia and gout, second edition [2], which was the latest edition when designing and conducting the trials. The guideline was revised in 2018 [1]; however, this paper describes based on the second edition. The guideline classified hyperuricemia into overproduction type, underexcretion type, and combined type, each, respectively, accounting for hyperuricemia in approximately $12 \%, 60 \%$, and $25 \%$ of patients [2]. Moreover, the guideline recommended drug selection based on the classification of hyperuricemia. Drug therapies for hyperuricemia 
are broadly divided into two classes: xanthine oxidoreductase inhibitors (XOIs), which reduce uric acid production, and uricosuric drugs, which increase urinary uric acid excretion [2]. In principle, it is proposed that uricosuric drugs should be used in underexcretion-type patients and XOIs should be used in overproduction-type patients [2]. However, it is reported that improvement in serum uric acid levels and symptoms of gout during treatment may influence the lifestyle of patients including dietary habits, thus leading to a change in the disease type [2]. This is probably because changes in body purine intake during diet therapy as well as changes in the total body uric acid pool resulting from treatment are responsible for changes in urinary uric acid excretion, one of the indices used for classification of hyperuricemia. Furthermore, due to the potential need to select drugs that diverge from the fundamental principle of the guidelines to avoid side effects, and reports that the rate of utilization of hyperuricemia classification by type is $35.6 \%$ in clinical practice [5], dotinurad may be administered to overproduction-type patients. When used in overproduction-type patients, a uricosuric agent is considered to excessively increase urinary uric acid excretion [2]. Moreover, it is reported that increased urinary uric acid excretion is associated with higher frequencies of urinary calculus formation [6]. In line with these considerations, the guideline recommends that uricostatic drugs should be used in overproduction-type patients with increased urinary uric acid excretion [1].

Dotinurad, a novel selective urate reabsorption inhibitor (SURI), reduces serum uric acid levels by selectively inhibiting urate transporter 1 (URAT1), which is expressed on the proximal renal tubules and is responsible for reabsorption of uric acid [7]. In contrast, benzbromarone, the most common uricosuric drug in Japan, has inhibitory effects not only on URAT1 but also on the organic anion transporter (OAT) 1 and OAT3, both of which are responsible for uric acid secretion in urine, and the ATP-binding cassette transporter G2 (ABCG2), which is responsible for uric acid secretion from the intestinal tract [7]. Both agents reduce serum uric acid levels by increasing urinary uric acid excretion; however, dotinurad, an SURI, is expected to be more efficient in reducing serum uric acid levels than benzbromarone, which inhibits multiple transporters.

Completed phase 2 studies of dotinurad demonstrated a dose-dependent serum uric acid lowering effect and a favorable safety profile [NCT\#02344862, NCT\#02416167]. In consideration of the possibility that dotinurad, a drug that reduces uric acid levels by stimulating uric acid excretion, would be used in overproduction-type patients, we conducted a clinical pharmacology study to compare the pharmacodynamics and safety of dotinurad between overproduction-type and underexcretion-type under hospitalized control conditions [NCT\#02837198]. In previous pharmacological studies, diet and lifestyle were controlled and these effects on urinary uric acid exclusion were eliminated; the results showed no significant differences between the two types in terms of percent change in serum uric acid level, amount of urinary uric acid excretion, and safety of dotinurad. On the other hand, the present study was conducted in outpatients to reflect the patient's actual diet and lifestyle because we consider it necessary to evaluate the pharmacodynamics and safety of dotinurad under conditions similar to those in actual clinical practice.

\section{Methods}

\section{Study design}

This was a multicenter, open-label, forced titration, clinical pharmacology study conducted at 10 medical institutions in Japan.

\section{Inclusion and exclusion criteria}

The inclusion criteria included the following: male patients aged 20 years and older at the time of informed consent; a serum uric acid level on the first day of the run-in period $\geq 7.0 \mathrm{mg} / \mathrm{dL}$ (patients with a history of gout or with gouty tophi), $\geq 8.0 \mathrm{mg} / \mathrm{dL}$ (patients with any of hypertension, diabetes mellitus, and metabolic syndrome under treatment or follow-up), or $\geq 9.0 \mathrm{mg} / \mathrm{dL}$ (patients without any of the above conditions under treatment or follow-up) in reference to the Japanese guideline [2]; patients who were classified as the overproduction or underexcretion type on the first day of the run-in period; outpatients; and patients who appropriately completed a 24-h urine collection on the final day of the run-in period. Incidentally, females were not included in this study because female had a menstrual cycle, it was considered difficult to implement appropriate urine collection, and many hyperuricemia patients were males [1].

The exclusion criteria included the following: patients with unresolved gouty arthritis in the 14 days before the day of assignment/registration; patients who used any serum uric acid-lowering agent during the specified period between the first day of the run-in period and the day of assignment/registration; patients who changed the dosing regimen of any drug that may affect serum uric acid levels between 14 days before the first day of the run-in period and the day of assignment/registration; patients with a history of allergy to potassium citrate/sodium citrate hydrate combination preparations; patients with a history of urinary calculus or in whom ultrasound or plain radiography of the abdomen on the start day of the run-in period revealed a urinary calculus; patients who previously received dotinurad; patients with $\mathrm{HbA} 1 \mathrm{c} \geq 8.4 \%$; patients with serum alanine aminotransferase (AST) and/or aspartate aminotransferase $\left(\right.$ ALT) $\geq 100 \mathrm{IU} / \mathrm{L}$, eGFR $<30 \mathrm{~mL} / \mathrm{min} / 1.73 \mathrm{~m}^{2}$, 
systolic blood pressure $\geq 180 \mathrm{mmHg}$ or diastolic blood pressure $\geq 110 \mathrm{mmHg}$ on the first day of the run-in period; and patients whose participation in the study was considered unsuitable in the opinion of the investigator.

Patients who were on uric acid-lowering medication underwent at least 28 days of washout after informed consent. Patients who were on medication that could affect serum uric acid levels or safety underwent at least 14 days of washout after informed consent.

\section{Treatment}

Figure 1 shows the study scheme. Prior to initiation of any study-related procedures, written informed consent was obtained from all patients. Patients were assigned to either the overproduction group or the underexcretion group at a 1:1 ratio based on the classification of hyperuricemia.

The study adopted dose titration to minimize the risk of gouty attacks due to a rapid fall in serum uric acid levels [1]. Dotinurad was administered at a starting dose of $0.5 \mathrm{mg} /$ day for two weeks and then at 1,2, and $4 \mathrm{mg} /$ day for four weeks each (a total of 14 weeks of treatment).

During the study, thirteen 24-h urine collections were conducted for pharmacodynamic evaluation (Fig. 1): two days before treatment at each dose, on the day and the day after starting treatment at each dose, and on the day before the end of treatment. A portable urine sampling container URINEMATE®-P (Sumitomo Bakelite Co., Ltd.) was provided to each patient, so that they could easily collect urine samples on their own, without mistakes.

To minimize the risk of urinary calculus formation associated with increased urinary uric acid excretion, patients concomitantly received urine alkalization therapy with

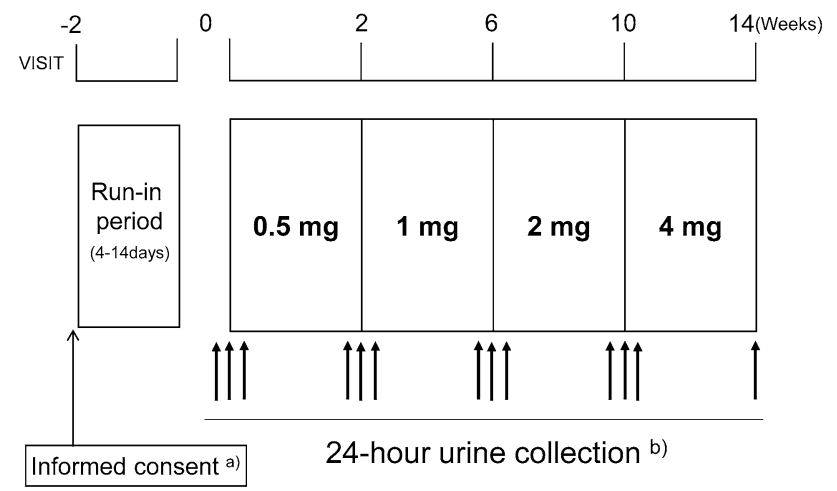

Fig. 1 Study scheme. a Patients underwent at least 28 days (on uric acid lowering medication) or 14 days (on medication that could affect efficacy evaluation or safety) of washout after informed concent, then started the run-in period. b During the study, thirteen 24-h urine collections were conducted for pharmacodynamic evaluation: 2 days before treatment at each dose, on the day and the day after starting treatment at each dose, and on the day before the end of treatment potassium citrate/sodium citrate hydrate combination preparations during the treatment period, unless the investigator decided that it was unnecessary.

\section{Hyperuricemia classification}

On the first day of the run-in period, a blood sample and a 60-min urine sample were collected and urinary extraction of uric acid $\left[E_{\mathrm{UA}}(\mathrm{mg} / \mathrm{kg} / \mathrm{h})\right]$ and uric acid clearance $\left[C_{\mathrm{UA}}\right.$ $(\mathrm{mL} / \mathrm{min})]$ were determined to identify the patient's disease type according to the following criteria: (i) overproduction type, $E_{\mathrm{UA}}>0.51$ and $C_{\mathrm{UA}} \geq 7.3$; (ii) underexcretion type, $E_{\mathrm{UA}}<0.48$ or $C_{\mathrm{UA}}<7.3$; (iii) combined type, $E_{\mathrm{UA}}>0.51$ and $C_{\mathrm{UA}}<7.3$; and (iv) normal type, $0.48 \leq E_{\mathrm{UA}} \leq 0.51$ and $C_{\mathrm{UA}} \geq 7.3$. Patients classified as the combined or normal type were excluded from the study. The hyperuricemic type was determined in accordance with the then current Japanese guideline for the management of hyperuricemia and gout [2], when the study was planned.

\section{Pharmacodynamic evaluations}

The primary endpoint was urinary uric acid excretion at 13 time points when 24-h urine samples were collected. The secondary endpoints were urinary uric acid/urinary creatinine levels at the same 13 time points and serum uric acid levels at selected time points (Weeks 2, 6, 10, and 14 and at the end of treatment).

\section{Safety evaluations}

The investigator evaluated adverse events (AEs) and safety based on vital signs, 12-lead electrocardiography, laboratory tests, and physical examination. AEs were coded by the System Organ Class and Preferred Term (MedDRA version 21.0) and the causal relationship to the study drug, and the severity and seriousness of each event were evaluated. An adverse drug reaction (ADR) was defined as an AE that was considered to be related to the study drug.

\section{Statistical analyses}

The target number of patients was determined to be 12 patients per group, in consideration of the feasibility of the study and a necessary sample size for pharmacodynamic assessment.

Pharmacodynamic analyses were performed on the full analysis set (FAS) consisting of patients who received at least one dose of the study drug and had at least one pharmacodynamic parameter evaluated after treatment.

The summary statistics of urinary uric acid excretion as the primary endpoint, and the summary statistics of 
secondary endpoints, namely, urinary uric acid/urinary creatinine levels and serum uric acid levels were also calculated for each group and their changes were plotted over time.

Safety analyses were performed on the safety population (SP) consisting of patients who received at least one dose of the study drug and had evaluable safety information after treatment. The number and proportion of patients with AEs and the number of AEs were calculated and tabulated. SAS software, version 9.3 (SAS Institute, Cary, NC, USA), was used in statistical analyses.

\section{Results}

\section{Patient flowcharts and baseline characteristics}

In this study, 57 patients were screened and 31 were excluded. Common reasons for exclusion were failure to meet the inclusion criteria and falling under exclusion criteria. The remaining 26 patients were assigned to either the overproduction group $(n=13)$ or the underexcretion group $(n=13)$. Eight patients in the overproduction group and five in the underexcretion group completed the study, and the remaining patients discontinued the study for the same reason: they all met a withdrawal criterion that urinary uric acid excretion on the day after starting treatment at each dose is $\geq 15 \%$ greater than that on the day of starting treatment at each dose (Fig. 2).

The FAS and SP comprised 13 patients each in the overproduction and underexcretion groups. (Fig. 2).

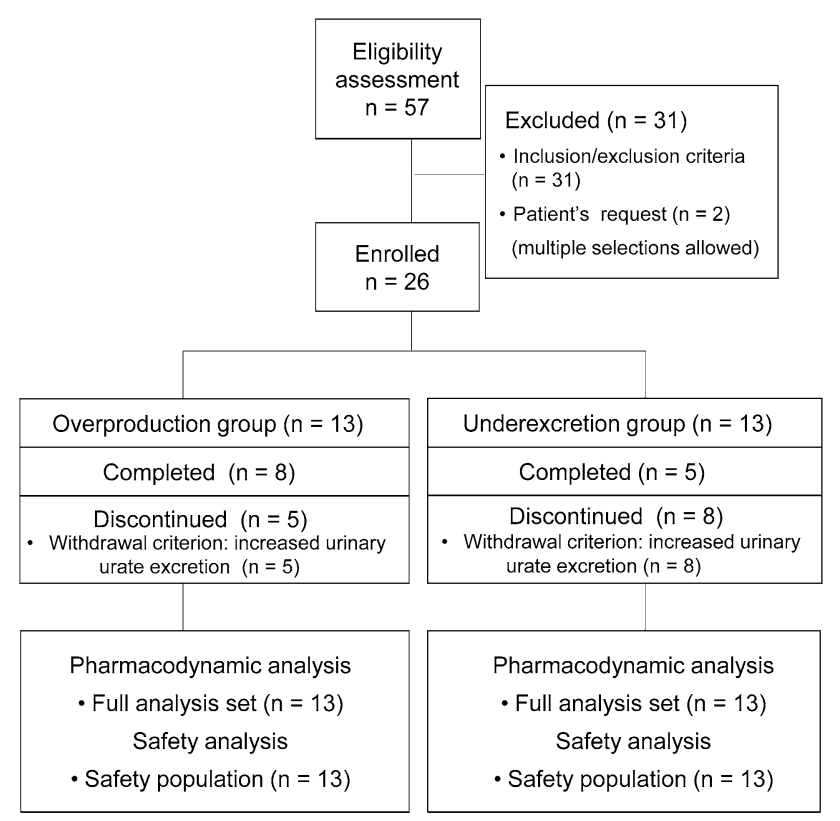

Fig. 2 Flowcharts of patients in this study
The demographic and other baseline characteristics in the FAS were as follows: mean ages of 50.5 and 61.6 years; mean urinary uric acid excretion of 648.84 and $609.58 \mathrm{mg}$; mean eGFR of 79.5 and $66.5 \mathrm{~mL} / \mathrm{min} / 1.73 \mathrm{~m}^{2}$; and the percentage of patients with a history of gouty arthritis of 100.0 and $61.5 \%$; mean Urinary uric acid/Creatinine ratio of 0.457 and 0.411 , in the overproduction and underexcretion groups, respectively. No relevant differences in the other characteristics were noted between the groups. The mean serum uric acid level before treatment was $8.31 \mathrm{mg} / \mathrm{dL}$ in the overproduction group and $8.29 \mathrm{mg} / \mathrm{dL}$ in the underexcretion group (Table 1).

\section{Pharmacodynamics}

\section{Primary endpoint}

The urinary uric acid excretion (mean \pm standard deviation [SD]) before treatment with $0.5 \mathrm{mg}$, on the day treatment started, on the day after starting treatment, and before dose titration to $1 \mathrm{mg}$ was $648.84 \pm 111.84,874.12 \pm 222.98$, $829.48 \pm 246.84$, and $801.65 \pm 177.50 \mathrm{mg}$, respectively, in the overproduction group and $609.58 \pm 244.36$, $864.21 \pm 371.25,788.88 \pm 310.64$, and $669.13 \pm 99.44 \mathrm{mg}$, respectively, in the underexcretion group. There was no meaningful difference between the groups in terms of changes in urinary uric acid excretion from before treatment to the end of treatment with $0.5 \mathrm{mg}$. During dose titration, a transient increase followed by a decrease in urinary uric acid excretion, as seen at the start of treatment, was observed in both groups. A similar pattern of changes was observed when the dose was titrated to 1,2 , and $4 \mathrm{mg}$. In both groups, urinary uric acid excretion transiently increased and then declined to approximately $800 \mathrm{mg}$ (Table 2, Fig. 3). The difference of urinary uric acid excretion between before treatment and end of treatment was $+171.49 \mathrm{mg}$ in the overproduction group, and $+120.10 \mathrm{mg}$ in the underexcretion group.

\section{Secondary endpoints}

The urinary uric acid/urinary creatinine ratio (mean \pm SD) before treatment with $0.5 \mathrm{mg}$, on the day treatment started, on the day after starting treatment, and before dose titration to $1 \mathrm{mg}$ was serum $0.457 \pm 0.076,0.611 \pm 0.125$, $0.578 \pm 0.086$, and $0.597 \pm 0.173 \mathrm{mg} / \mathrm{dL}$, respectively, in the overproduction group and $0.411 \pm 0.067,0.562 \pm 0.102$, $0.532 \pm 0.116$, and $0.434 \pm 0.057 \mathrm{mg} / \mathrm{dL}$, respectively, in the underexcretion group. During dose titration, a transient increase followed by a decrease in urinary uric acid/creatinine ratio, as seen at the start of treatment, was observed in both groups (Table 3, Fig. 4). This tendency was similar to that for urinary uric acid excretion (primary endpoint). The 
Table 1 Summary of the baseline characteristics

\begin{tabular}{|c|c|c|c|c|c|c|}
\hline Characteristics & & & $\begin{array}{l}\text { Overproduction } \\
\text { group }(n=13)\end{array}$ & $\begin{array}{l}\text { Underexcretion } \\
\text { group }(\mathrm{n}=13)\end{array}$ & Total $(n=26)$ & P value ${ }^{c}$ \\
\hline \multirow[t]{3}{*}{ Sex } & No of patients $(\%)$ & Male & $13(100.0)$ & $13(100.0)$ & $26(100.0)$ & - \\
\hline & & & & & & - \\
\hline & & Female & $0(0.0)$ & $0(0.0)$ & $0(0.0)$ & \\
\hline Age (years) & Mean \pm SD & & $50.5 \pm 10.3$ & $61.6 \pm 10.0$ & $56.0 \pm 11.4$ & $0.012^{\mathrm{d}}$ \\
\hline Height $(\mathrm{cm})$ & Mean \pm SD & & $169.00 \pm 6.10$ & $169.40 \pm 6.96$ & $169.20 \pm 6.41$ & $1.000^{\mathrm{d}}$ \\
\hline Body weight (kg) & Mean \pm SD & & $72.62 \pm 14.63$ & $76.14 \pm 12.34$ & $74.38 \pm 13.38$ & $0.317^{\mathrm{d}}$ \\
\hline $\operatorname{BMI}\left(\mathrm{kg} / \mathrm{m}^{2}\right)$ & Mean \pm SD & & $25.43 \pm 5.06$ & $26.44 \pm 3.32$ & $25.94 \pm 4.23$ & $0.249^{\mathrm{d}}$ \\
\hline Urinary uric acid excretion (mg) & Mean \pm SD & & $648.84 \pm 111.84$ & $609.58 \pm 244.36$ & $629.21 \pm 187.26$ & $0.118^{\mathrm{d}}$ \\
\hline Serum uric acid level (mg/dL) & Mean \pm SD & & $8.31 \pm 0.56$ & $8.29 \pm 0.60$ & $8.30 \pm 0.57$ & $0.877^{\mathrm{d}}$ \\
\hline $\mathrm{eGFR}\left(\mathrm{mL} / \mathrm{min} / 1.73 \mathrm{~m}^{2}\right)^{\mathrm{a}}$ & Mean \pm SD & & $79.5 \pm 11.1$ & $66.5 \pm 10.7$ & $73.0 \pm 12.6$ & $0.009^{\mathrm{d}}$ \\
\hline Urinary uric acid/Creatinine ratio & Mean \pm SD & & $0.457 \pm 0.076$ & $0.411 \pm 0.067$ & $0.434 \pm 0.074$ & $0.118^{\mathrm{d}}$ \\
\hline \multirow[t]{2}{*}{ Treatment history } & No of patients $(\%)$ & No & $3(23.1)$ & $3(23.1)$ & $6(23.1)$ & $1.000^{\mathrm{e}}$ \\
\hline & & Yes & $10(76.9)$ & $10(76.9)$ & $20(76.9)$ & \\
\hline \multirow[t]{2}{*}{ History of gouty arthritis } & No of patients (\%) & No & $0(0.0)$ & $5(38.5)$ & $5(19.2)$ & $0.039^{\mathrm{e}}$ \\
\hline & & Yes & $13(100.0)$ & $8(61.5)$ & $21(80.8)$ & \\
\hline \multirow[t]{2}{*}{ Gouty Tophi } & No of patients (\%) & No & $13(100.0)$ & $13(100.0)$ & $26(100.0)$ & - \\
\hline & & Yes & $0(0.0)$ & $0(0.0)$ & $0(0.0)$ & \\
\hline \multirow[t]{2}{*}{ Drinking habit ${ }^{\mathrm{b}}$} & No of patients (\%) & No & $4(30.8)$ & $3(23.1)$ & $7(26.9)$ & $1.000^{\mathrm{e}}$ \\
\hline & & Yes & $9(69.2)$ & $10(76.9)$ & $19(73.1)$ & \\
\hline
\end{tabular}

a eGFR $\left(\mathrm{mL} / \mathrm{min} / 1.73 \mathrm{~m}^{2}\right)=194 \times$ Serum creatinine ${ }^{-1.094} \times$ Age $^{-0.287}$

${ }^{b}$ Definition of drinking habit: consumption of alcohol on more than 3 days of the week and consumption of more than $500 \mathrm{~mL}$ of beer or $60 \mathrm{~mL}$ of whisky in a day

${ }^{\mathrm{c}}$ Two-sided significance level of $15 \%$,

${ }^{\mathrm{d}}$ Wilcoxon rank-sum test

${ }^{\text {e}}$ Fisher's exact test

urinary uric acid/creatinine ratio at end of treatment was 1.28 times in the overproduction group and 1.21 times in the underexcretion group compared to those at the start of treatment.

No relevant difference in changes in serum uric acid levels was noted between the groups at any time points. The serum uric acid level (mean \pm SD) in the overproduction and underexcretion groups was $3.19 \pm 0.78$ and $3.58 \pm 0.99 \mathrm{mg} / \mathrm{dL}$, respectively, at Week 14 and $3.98 \pm 1.55$ and $3.93 \pm 1.28 \mathrm{mg} /$ dL, respectively, at the end of treatment (Fig. 5).

\section{Safety}

The incidence of AEs in the overproduction and underexcretion groups was 15.4 and $38.5 \%$, respectively. The incidence of ADRs in the overproduction and underexcretion groups was 7.7 and $15.4 \%$, respectively. The ADRs included 'low density lipoprotein increased' in the overproduction group, and 'musculoskeletal discomfort' and 'oedema peripheral' in the underexcretion group (each with one event in a single patient). In either group, no AEs occurred in more than one subject, and all reported AEs were mild in severity. Serious adverse events, AEs leading to treatment discontinuation
(Table 4), gouty arthritis, or urinary calculus were not reported in either group.

\section{Discussion}

Dotinurad, an SURI, reduces serum uric acid levels by increasing urinary uric acid excretion. Researchers report a positive correlation between urinary uric acid excretion and the prevalence of urinary calculi in patients with gout [6]. The use of uricosuric agents in hyperuricemic patients of the overproduction type is thought to cause a marked increase in urinary uric acid excretion [2], though it has yet to be proven in randomized controlled studies. However, uricosuric drugs are assumed to be prescribed to patients with hyperuricemia of the overproduction type in actual clinical practice. One of the reasons is a reported change in the disease type during treatment. In our previous inpatient study, some patients experienced a change in their disease type from the overproduction type to the underexcretion type during screening period of the study [NCT\#02837198]. In light of these findings, evaluation of the pharmacodynamics and safety of dotinurad in each hyperuricemia type has great 
Table 2 Urinary uric acid excretion at each time point

\begin{tabular}{|c|c|c|c|c|c|}
\hline & \multirow[t]{3}{*}{ Time point } & \multicolumn{4}{|c|}{ Urinary uric acid excretion (mg) } \\
\hline & & \multirow[t]{2}{*}{ No. of patients } & \multirow[t]{2}{*}{ Mean \pm SD } & \multicolumn{2}{|c|}{ Two-sided 95\% CI for the mean } \\
\hline & & & & Lower limit & Upper limit \\
\hline \multicolumn{6}{|c|}{ Overproduction group } \\
\hline \multirow[t]{3}{*}{$0.5 \mathrm{mg}$} & Before treatment & 13 & $648.84 \pm 111.84$ & 581.25 & 716.42 \\
\hline & Day treatment started & 13 & $874.12 \pm 222.98$ & 739.37 & 1008.86 \\
\hline & Day after starting treatment & 13 & $829.48 \pm 246.84$ & 680.32 & 978.64 \\
\hline \multirow[t]{3}{*}{$1 \mathrm{mg}$} & Before dose increase & 11 & $801.65 \pm 177.50$ & 682.41 & 920.90 \\
\hline & Day of dose increase & 11 & $855.10 \pm 290.36$ & 660.03 & 1050.17 \\
\hline & Day after dose increase & 11 & $798.82 \pm 229.25$ & 644.81 & 952.83 \\
\hline \multirow[t]{3}{*}{$2 \mathrm{mg}$} & Before dose increase & 8 & $796.61 \pm 143.45$ & 676.69 & 916.54 \\
\hline & Day of dose increase & 8 & $959.53 \pm 190.56$ & 800.21 & 1118.84 \\
\hline & Day after dose increase & 8 & $825.84 \pm 211.81$ & 648.76 & 1002.91 \\
\hline \multirow[t]{4}{*}{$4 \mathrm{mg}$} & Before dose increase & 8 & $752.63 \pm 174.31$ & 606.90 & 898.35 \\
\hline & Day of dose increase & 8 & $941.95 \pm 180.68$ & 790.90 & 1093.00 \\
\hline & Day after dose increase & 8 & $803.94 \pm 222.57$ & 617.86 & 990.01 \\
\hline & End of treatment & 8 & $820.33 \pm 135.34$ & 707.18 & 933.47 \\
\hline \multicolumn{6}{|c|}{ Underexcretion group } \\
\hline \multirow[t]{3}{*}{$0.5 \mathrm{mg}$} & Before treatment & 13 & $609.58 \pm 244.36$ & 461.92 & 757.25 \\
\hline & Day treatment started & 13 & $864.21 \pm 371.25$ & 639.86 & 1088.55 \\
\hline & Day after starting treatment & 13 & $788.88 \pm 310.64$ & 601.16 & 976.59 \\
\hline \multirow[t]{3}{*}{$1 \mathrm{mg}$} & Before dose increase & 9 & $669.13 \pm 99.44$ & 592.70 & 745.57 \\
\hline & Day of dose increase & 9 & $798.77 \pm 149.10$ & 684.16 & 913.38 \\
\hline & Day after dose increase & 9 & $761.02 \pm 186.76$ & 617.47 & 904.58 \\
\hline \multirow[t]{3}{*}{$2 \mathrm{mg}$} & Before dose increase & 8 & $778.68 \pm 354.69$ & 482.15 & 1075.20 \\
\hline & Day of dose increase & 8 & $843.11 \pm 309.42$ & 584.43 & 1101.79 \\
\hline & Day after dose increase & 8 & $843.79 \pm 269.59$ & 618.40 & 1069.17 \\
\hline \multirow[t]{4}{*}{$4 \mathrm{mg}$} & Before dose increase & 7 & $801.60 \pm 374.43$ & 455.31 & 1147.89 \\
\hline & Day of dose increase & 7 & $826.63 \pm 248.06$ & 597.21 & 1056.04 \\
\hline & Day after dose increase & 7 & $793.29 \pm 303.07$ & 512.99 & 1073.58 \\
\hline & End of treatment & 5 & $729.68 \pm 124.60$ & 574.97 & 884.39 \\
\hline
\end{tabular}

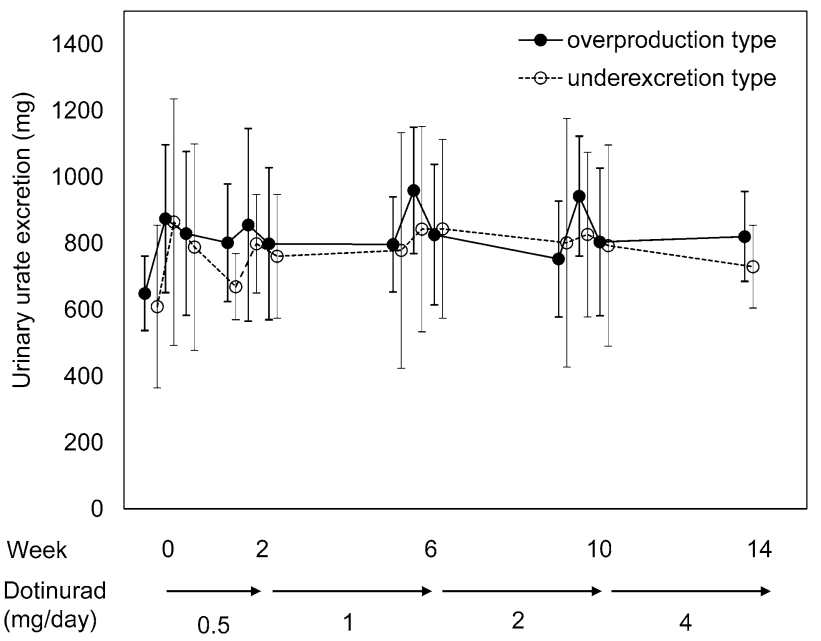

Fig. 3 Time course of urinary uric acid excretion. Error bars indicate the standard deviation (SD) significance, under the assumption that the dotinurad may be used irrespective of the hyperuricemia type.

This study evaluated the pharmacodynamics and safety of dotinurad administered for 14 weeks in hyperuricemia outpatients with or without gout of the overproduction and underexcretion types.

Some points should be considered before interpreting the results of the study. Of 26 patients enrolled, 13 (5 in the overproduction group and 8 in the underexcretion group) discontinued the study for the same reason: they all met a withdrawal criterion that urinary uric acid excretion on the day after starting treatment at each dose (dose increase) is $\geq 15 \%$ greater than that on the day of starting treatment at each dose (dose increase). This criterion was used in this study to minimize the risk of urinary calculus formation in patients in whom an increase in urinary uric acid excretion was noted on the day after starting treatment, on the basis 
Table 3 Urinary uric acid/creatinine ratio at each time point

\begin{tabular}{|c|c|c|c|c|c|}
\hline & \multirow[t]{3}{*}{ Time point } & \multicolumn{4}{|c|}{ Urinary uric acid/creatinine ratio } \\
\hline & & \multirow[t]{2}{*}{ No. of patients } & \multirow[t]{2}{*}{ Mean \pm SD } & \multicolumn{2}{|c|}{ Two-sided $95 \%$ CI for the mean } \\
\hline & & & & Lower limit & Upper limit \\
\hline \multicolumn{6}{|c|}{ Overproduction group } \\
\hline \multirow[t]{3}{*}{$0.5 \mathrm{mg}$} & Before treatment & 13 & $0.457 \pm 0.076$ & 0.411 & 0.503 \\
\hline & Day treatment started & 13 & $0.611 \pm 0.125$ & 0.536 & 0.687 \\
\hline & Day after starting treatment & 13 & $0.578 \pm 0.086$ & 0.526 & 0.630 \\
\hline \multirow[t]{3}{*}{$1 \mathrm{mg}$} & Before dose increase & 11 & $0.597 \pm 0.173$ & 0.481 & 0.713 \\
\hline & Day of dose increase & 11 & $0.658 \pm 0.182$ & 0.535 & 0.780 \\
\hline & Day after dose increase & 11 & $0.569 \pm 0.103$ & 0.500 & 0.639 \\
\hline \multirow[t]{3}{*}{$2 \mathrm{mg}$} & Before dose increase & 8 & $0.574 \pm 0.138$ & 0.458 & 0.689 \\
\hline & Day of dose increase & 8 & $0.760 \pm 0.257$ & 0.545 & 0.975 \\
\hline & Day after dose increase & 8 & $0.671 \pm 0.200$ & 0.504 & 0.838 \\
\hline \multirow[t]{4}{*}{$4 \mathrm{mg}$} & Before dose increase & 8 & $0.580 \pm 0.229$ & 0.388 & 0.771 \\
\hline & Day of dose increase & 8 & $0.711 \pm 0.204$ & 0.541 & 0.881 \\
\hline & Day after dose increase & 8 & $0.589 \pm 0.102$ & 0.503 & 0.674 \\
\hline & End of treatment & 8 & $0.583 \pm 0.174$ & 0.437 & 0.728 \\
\hline \multicolumn{6}{|c|}{ Underexcretion group } \\
\hline \multirow[t]{3}{*}{$0.5 \mathrm{mg}$} & Before treatment & 13 & $0.411 \pm 0.067$ & 0.370 & 0.451 \\
\hline & Day treatment started & 13 & $0.562 \pm 0.102$ & 0.501 & 0.624 \\
\hline & Day after starting treatment & 13 & $0.532 \pm 0.116$ & 0.462 & 0.602 \\
\hline \multirow[t]{3}{*}{$1 \mathrm{mg}$} & Before dose increase & 9 & $0.434 \pm 0.057$ & 0.390 & 0.477 \\
\hline & Day of dose increase & 9 & $0.552 \pm 0.098$ & 0.477 & 0.628 \\
\hline & Day after dose increase & 9 & $0.487 \pm 0.070$ & 0.433 & 0.540 \\
\hline \multirow[t]{3}{*}{$2 \mathrm{mg}$} & Before dose increase & 8 & $0.501 \pm 0.095$ & 0.421 & 0.580 \\
\hline & Day of dose increase & 8 & $0.573 \pm 0.151$ & 0.447 & 0.699 \\
\hline & Day after dose increase & 8 & $0.559 \pm 0.094$ & 0.480 & 0.638 \\
\hline \multirow[t]{4}{*}{$4 \mathrm{mg}$} & Before dose increase & 7 & $0.513 \pm 0.096$ & 0.424 & 0.603 \\
\hline & Day of dose increase & 7 & $0.610 \pm 0.052$ & 0.562 & 0.658 \\
\hline & Day after dose increase & 7 & $0.528 \pm 0.100$ & 0.436 & 0.620 \\
\hline & End of treatment & 5 & $0.498 \pm 0.049$ & 0.437 & 0.559 \\
\hline
\end{tabular}

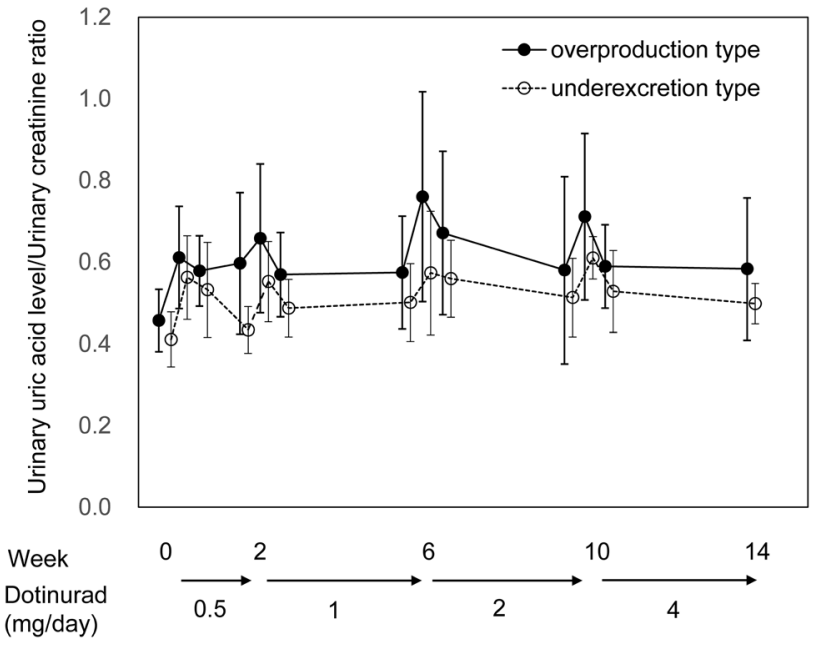

Fig. 4 Time course of Urinary uric acid/Creatinine ratio. Error bars indicate the standard deviation (SD) of the findings in the inpatient study that urinary uric acid excretion increased on the day on which treatment started, and decreased on the following day. A urinary calculus may be caused not only by increased urinary uric acid excretion but also by decreased urine output/insufficient water intake and persistently acid urine [1]. These factors elevate urinary uric acid levels or lower the solubility of urinary uric acid, thereby triggering the deposition of uric acid crystals in the urinary tract. In this study, urine output in both groups was not meaningfully different (Supplement 1).

Individual review of changes in urinary uric acid excretion over time revealed that urinary uric acid excretion in patients who discontinued, with some exceptions, was not biased toward higher levels compared to patients who completed the study (Fig. 6). Urinary uric acid excretion at discontinuation substantially exceeded $1000 \mathrm{mg}$ in one patient each in the two groups. To evaluate the risk of urinary 


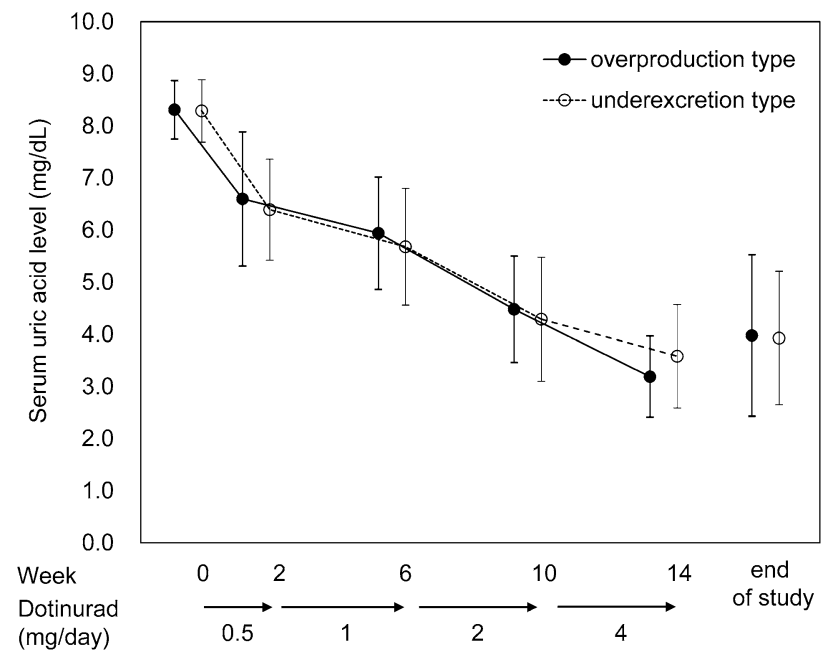

Fig. 5 Time course of the serum uric acid level. Error bars indicate the standard deviation (SD)

calculus formation from another point of view, urinary uric acid levels at discontinuation were reviewed in these two patients. They were 40.1 and $46.4 \mathrm{mg} / \mathrm{dL}$, which did not meaningfully differ from those patients who completed the study. Urine output at discontinuation in both patients (3350 and $3150 \mathrm{~mL}$ ) substantially exceeded $1500 \mathrm{~mL}$, the upper limit of daily urine output in normal individuals [8]. Based on these findings, polyuria could explain the increased urinary uric acid excretion observed in both patients.

The two patients had preexisting polyuria, which was likely to be inherent. The other 11 patients included those who experienced an increase in urinary uric acid excretion for three days in a row (before treatment, the day treatment started, after starting treatment) but their urinary uric acid excretion levels were not high; and those who experienced a decline in urinary uric acid excretion on the day of starting treatment followed by an increase on the following day, thus meeting the withdrawal criterion. Withdrawals due to this criterion occurred in both hyperuricemic types.
The results of the study showed no relevant differences in changes in urinary uric acid excretion (primary endpoint) between the overproduction and underexcretion types. Urinary uric acid excretion increased on the day of starting treatment (dose increase) and then decreased on the following day, as observed in the inpatient study [NCT\#02837198]. Unlike the inpatient study, the present study in outpatients tended to show a greater variation between patients. This may be explained by greater differences in dietary habit and lifestyle among outpatients.

No relevant differences were observed in the secondary endpoints between the overproduction and underexcretion types. Dotinurad reduced serum uric acid levels similarly in both groups.

During the study, the dose was titrated to $4 \mathrm{mg}$, the assumed maximum dose of dotinurad. No noteworthy safety concerns arose in each group.

There are some limitations of this study. Firstly, this study was an exploratory study conducted in a small number of patients. Secondary, there are no data available regarding extended treatment with dotinurad in the patients withdrawn from the study. Hence, it is practically impossible to draw a definite conclusion solely from the results of the study regarding changes in urinary uric acid excretion as well as the risk of urinary calculus formation for each hyperuricemic type; however, the results in the study do not indicate, from the perspective of urinary uric acid excretion, that dotinurad therapy clearly increases the risk of urinary calculus formation in overproduction-type patients. Any increase in urinary uric acid excretion after dosing with dotinurad was transient, as observed in the previous inpatient study. Combining the results of the two studies, changes in urinary uric acid excretion after dotinurad administration do not differ in a clinically meaningful way between the overproduction and underexcretion types, and no urinary calculus was found in any patient in either group in this study.

Finally, the 2018 revised guidelines (Third Edition) changed the hyperuricemia classification and defined overproduction type as renal overload type. The renal overload type is further categorized as either overproduction or
Table 4 Summary of adverse events

\begin{tabular}{|c|c|c|c|c|c|c|}
\hline & \multicolumn{3}{|c|}{ Overproduction type } & \multicolumn{3}{|c|}{ Underexcretion type } \\
\hline & \multicolumn{3}{|l|}{$(n=13)$} & \multicolumn{3}{|l|}{$(n=13)$} \\
\hline & $\begin{array}{l}\text { No. of } \\
\text { patients }\end{array}$ & Incidence $(\%)$ & No. of events & $\begin{array}{l}\text { No. of } \\
\text { patients }\end{array}$ & Incidence (\%) & No. of events \\
\hline AEs & 2 & $(15.4)$ & 4 & 5 & $(38.5)$ & 5 \\
\hline ADRs & 1 & $(7.7)$ & 1 & 2 & ( 15.4$)$ & 2 \\
\hline SAEs excluding deaths & 0 & $(0.0)$ & 0 & 0 & $(0.0)$ & 0 \\
\hline $\begin{array}{l}\text { AEs leading to treat- } \\
\text { ment discontinuation }\end{array}$ & 0 & $(0.0)$ & 0 & 0 & $(0.0)$ & 0 \\
\hline
\end{tabular}



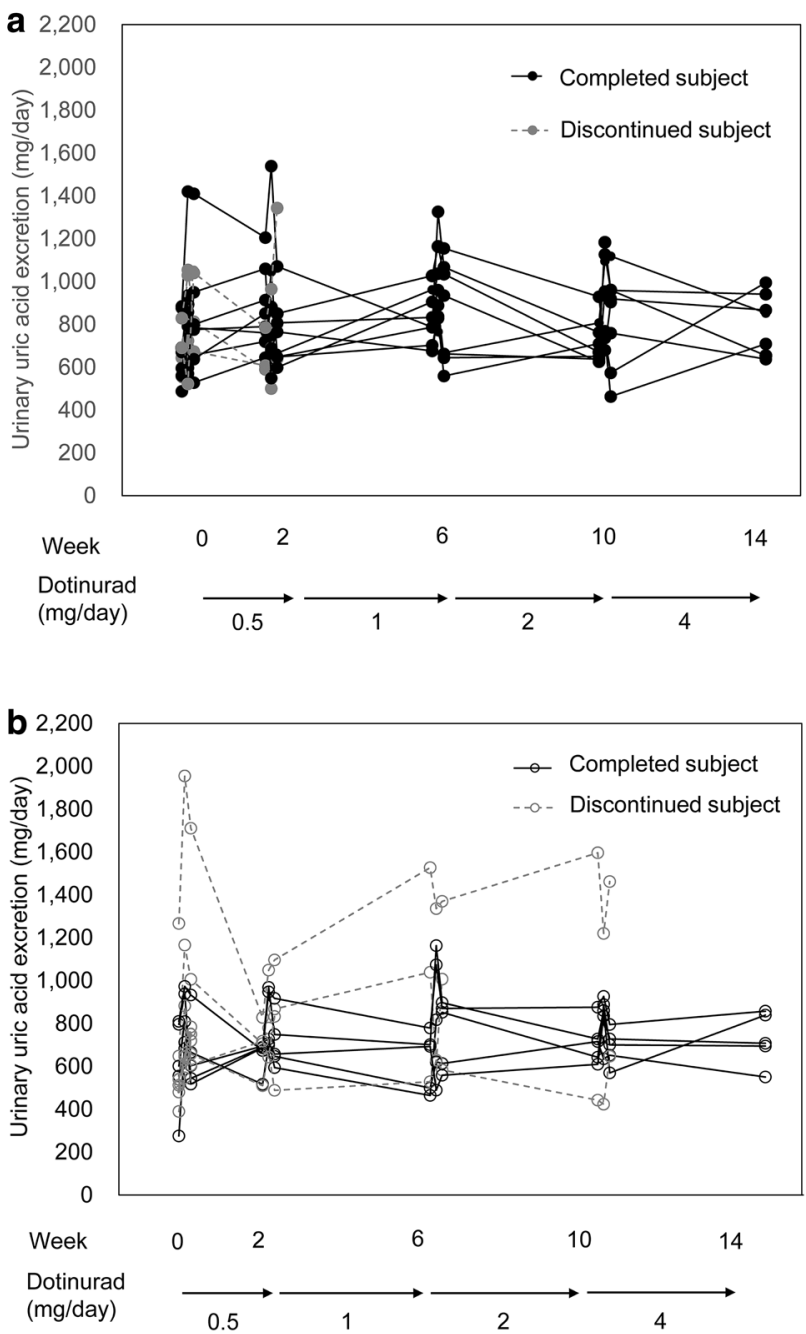

Fig. 6 Individual data on urinary uric acid excretion. a Overproduction type. b Underexcretion type

extra-renal underexcretion type, but the criteria for which are not defined; so, the breakdown of the overproduced group in this study is unknown. The consequences of overproduction type need to be further investigated.

Acknowledgements This research was sponsored by Mochida pharmaceutical Co., Ltd. and Fuji Yakuhin Co., Ltd. We thank SunFlare Co., Ltd. for editorial assistance in preparing this manuscript.

\section{Compliance with ethical standards}

Conflict of interest TH was an advisor to Fuji Yakuhin Co., Ltd. regarding this study and received consultant and manuscript fees. KF and SK are employees of Mochida Pharmaceutical Co. Ltd.

Ethical standards This study was conducted according to the Declaration of Helsinki, International Committee on Harmonization Good Clinical Practice guideline, and applicable local laws and regulations. The protocol was approved by the regulatory authorities in Japan and by the ethics committees of study center. All patients provided written informed consent before starting any procedure in this study. The information of this study was registered with ClinicalTrials.gov (Identifier: NCT03375632).

Open access This article is distributed under the terms of the Creative Commons Attribution 4.0 international license (), which permits unrestricted use, distribution, and reproduction in any medium, provided you give appropriate credit to the original author(s) and the source, provide a link to the Creative Commons license, and indicate if changes were made.

Open Access This article is licensed under a Creative Commons Attribution 4.0 International License, which permits use, sharing, adaptation, distribution and reproduction in any medium or format, as long as you give appropriate credit to the original author(s) and the source, provide a link to the Creative Commons licence, and indicate if changes were made. The images or other third party material in this article are included in the article's Creative Commons licence, unless indicated otherwise in a credit line to the material. If material is not included in the article's Creative Commons licence and your intended use is not permitted by statutory regulation or exceeds the permitted use, you will need to obtain permission directly from the copyright holder. To view a copy of this licence, visit http://creativecommons.org/licenses/by/4.0/.

\section{References}

1. Japanese Society of Gout and Nucleic Acid Metabolism. Japanese guideline for the management of hyperuricemia and gout: 3rd edition. Tokyo: SHINDAN TO CHIRYO SHA, Inc.; 2018. p. 18-23, 56-9, 72-3, 114-7, 122-4.

2. Japanese Society of Gout and Nucleic Acid Metabolism. Japanese guideline for the management of hyperuricemia and gout: second edition. Osaka; Medical Review Co., Ltd; 2010. p30-1, p63-5, p83-7.

3. Marangella M. Uric acid elimination in the urine Pathophysiological implications. Contrib Nephrol. 2005;147:132-48.

4. Shimizu T, Kitada H, Umeyama M, et al. Novel evaluation of nephrolithiasis as a complication of gout: a cross-sectional study using helical computerized tomography. J Urol. 2013;189:1747-52.

5. Oikawa T, Goto Y, Sato K, et al. Post-marketing Surveillance of Benzbromarone (Urinorm ${ }^{\circledR}$ Tablets) - Incidences of adverse drug reactions and changes in laboratory values relating to safety and efficacy under routine drug usage.(in Japanese) Gout and Nucleic Acid Metabolism. 2011; 35:19-30.

6. Abou-Elela A. Epidemiology, pathophysiology, and management of uric acid urolithiasis: a narrative review. J Adv Res. 2017;8:513-27.

7. Taniguchi T, Ashizawa N, Matsumoto K, et al. Pharmacological evaluation of dotinurad, a selective urate reabsorption inhibitor. J Pharmacol Exp Ther. 2019. https://doi.org/10.1124/ jpet.119.259341.

8. Yuno T. Urine and feces inspection. In: Kanai M, supervisor. Okumura N, Tozuka M, Yatomi Y, editors. Kanai's Manual of Clinical Laboratory Medicine (Revised 34th Edition). Japan: KANEHARA \& CO., LTD.; 2015. pp.125

Publisher's Note Springer Nature remains neutral with regard to jurisdictional claims in published maps and institutional affiliations. 